\title{
Multihop Uncoordinated Cooperative Forwarding in Highly Dynamic Networks
}

\author{
Xuefei Zhang*, Guoqiang Mao ${ }^{\dagger \ddagger}$, Xiaofeng Tao*, Qimei Cui* and Baoling Liu* \\ * Beijing University of Posts and Telecommunications, China \\ $\dagger$ University of Technology Sydney, Australia \\ $\ddagger$ National ICT Australia (NICTA), Australia \\ Email: zhangxuefeikate@gmail.com, Guoqiang.Mao@uts.edu.au, \{taoxf, cuiqimei, blliu\}@bupt.edu.cn,
}

\begin{abstract}
This paper investigates cooperative forwarding in large highly dynamic wireless networks. Unlike traditional coordinated cooperative forwarding schemes that require a large amount of neighborhood discovery and coordination information to be exchanged before making the forwarding decision, this paper proposes an uncoordinated cooperative forwarding scheme where each node determines whether or not to forward a received packet independently based on a forwarding probability determined by its own location, the density of nodes in the network and the locations of the destination and the transmitter from which it receives the packet, without the costly or even impractical neighbor discovery and coordination process. Analytical results are derived on the successful transmission probability and the expected number of forwarding nodes involved in the cooperative forwarding process. On that basis, discussions are presented on the optimal forwarding probability design that meets a pre-designated successful transmission probability targ: et $^{*}$ using minimum number of forwarding nodes. Simulations are conducted to evaluate the performance of the proposed scheme.
\end{abstract}

Index Terms-highly dynamic networks, multihop, uncoordinated cooperative forwarding.

\section{INTRODUCTION}

This paper considers the problem of cooperative forwarding in large highly dynamic wireless networks, e.g. vehicular ad hoc networks (VANET) or mobile ad hoc networks (MANET). On a high level, the problem can be described as: when a node in a large highly dynamic wireless network overhears a packet belonging to a particular source-destination pair, with minimal information about its neighborhood and environment, how the node makes decision on whether it should collaborate to forward the packet?

Of course, if every node overhearing the packet forwards the packet with a high probability, the packet can be delivered to its destination with a high probability but it may cause a large number of redundant transmissions thereby wasting precious radio resources. On the other hand, if the forwarding probability is low, the packet may not eventually arrive at its destination. Therefore, tradeoff between three main factors are involved in the decision process: 1) the amount of information used in making the forwarding decision. The more information is used, the more overhead is incurred in collecting the information. It was reported that current military prototype MANFTs routinelv exnerience overhead on the order of even 99 percent of the end-to-end packet transmissions [1]. Therefore overhead involved in the cooperative forwarding decision is an important consideration; 2) the forwarding probability which determines the number of nodes (or equivalently transmissions) involved in the cooperative forwarding process; and 3) the successful transmission probability, i.e. the probability that the packet eventually arrives at its destination within a designated amount of time.

A major challenge for routing and forwarding in dynamic networks is that the nodes are constantly moving and the network topology is highly dynamic. Therefore the traditional layered approach, where the route between a source and its destination is determined before the actual data transfer, is unsuitable for dynamic networks. *There are two common approaches to end-to-end packet transmissions in highly dynamic networks: broadcast and cooperative communication. Broadcast remains to be the most reliable and possibly the most widely used approach for packet transmission in highly dynamic networks [2], [3] however it is well known to cause a large number of redundant transmissions and significant wastage in radio resources. Cooperative communication on the other hand allows additional nodes in the vicinity of the route that overhear the transmitted packet to assist in delivering the packet to its destination, leveraging the broadcast nature of the wireless medium to provide diversity against timevarying link fades and outages [4].

A common feature in existing cooperative techniques is the coordination required among the participating neighbors. These coordinations typically include the discovery of neighbors in the vicinity, the collection of channel information to these neighbors, and the selection of the best neighbor(s) whose cooperation will maximize the performance improvement [2], [4], [5], [6], [7]. For the well-known opportunistic routing schemes [8], coordination is required at every hop to decide the node that will serve as the packet's next hop towards the destination. It was reported in [1] that the coordination overhead may account for 99 percent of the end-to-end packet transmissions. Due to associated coordination overheads, existing cooperative communication methods are suitable mostly for mesh or sensor networks with static or relatively stable topologies. They are not useful when the topology is very dynamic, due to either a high velocity (e.g. vehicular networks) or a high density of the nodes (e.g. networks 
of mobile devices carried by people on a busy street or in a conference hall). In fact, in highly dynamics networks, the coordination overheads are incurred too frequently to be practical even just to maintain an up-to-date view of the neighbor topology, letting alone an up-to-date channel state information to the neighbor nodes.

Motivated by the above observations, in this paper we consider an uncoordinated cooperative forwarding scheme, where nodes overhearing a packet make forwarding decisions independently without prior coordination or measurement of real-time channel information to its neighbors, and even without being aware of their existence (apart from the transmitter of the packet). Furthermore, forwarding decision at each node is only based on the location of that node, the locations of the destination and the transmitter from which it receives the packet, and some limited prior statistical knowledge about the local environment, namely, the spatial distribution of the nodes and radio propagation characteristics. A major challenge in the uncoordinated cooperative forwarding scheme is the design of the forwarding probability that on the one hand minimizing the number of transmissions required to deliver the packet to its destination, and on the other hand guaranteeing a designated transmission success probability. In the literature, the forwarding probability has been chosen to be a predefined fixed value [9], a linear function of the distance between the transmitter and the receiver [10], or be determined jointly by the distance to the destination and nodes' spatial distribution [2]. In [4], theoretical analysis was presented on the successful transmission probability using three uncoordinated forwarding schemes in two-hop scenario where the source and the destination are at most two hops away. Reference [5] further obtained the optimal forwarding scheme in the two-hop scenario. Despite the above advances in the field, design of optimal uncoordinated forwarding scheme for multi-hop scenarios, backed by solid theoretical analysis, remains an open challenge. It is a focus of this paper to tackle the challenge.

More specifically, the main contributions of this paper are:

- Considering one-dimensional highly dynamic networks, with vehicular networks on a highway being typical examples, this paper proposes an uncoordinated cooperative forwarding scheme, where each node receiving the packet makes forwarding decisions independently of other nodes, using its own location, the locations of the destination and the transmitter from which it receives the packet, the spatial distribution of nodes and radio propagation characteristics only, without prior coordination with its neighbors and even without being aware of their existence.

- Performance of the proposed uncoordinated forwarding scheme is analyzed. For a pair of source and destination separated multiple hops away and with a known distance, the expected number of forwarding nodes and the successful transmission probability are obtained.

- On the basis of the analysis, discussions are presented on the optimal design of the forwarding probabil- ity function to meet a pre-designated target on the probability of successful transmission while using the minimum number of forwarding nodes.

- Simulations are conducted to validate the performance of the proposed uncoordinated cooperative forwarding scheme.

The technique and analysis presented in this paper can be useful for designing cooperative communication strategies in large and highly dynamic networks.

The rest of the paper is organized as follows. In Section II, we give an accurate definition of the network models, explain the design of the uncoordinated cooperative forwarding scheme and the problem formulation. Section III presents performance analysis of the proposed uncoordinated cooperative forwarding scheme and on that basis discusses the design of the optimal forwarding probability function. Section IV presents simulations and discussions. Finally, Section V concludes the paper.

\section{NETWORK MODEL}

In this paper, we consider a one-dimensional (1-D) dynamic network on a linear segment of $L$ with a single source-destination pair. The source and the destination are placed at the two ends of the segment. Without loss of generality, we assume that the source is located at the origin and the destination is located on $+x$ axis. All other nodes apart from the source and the destination are distributed on the segment following a homogeneous Poisson distribution with known density $\rho$. Note that the probability density function of the distance between a pair of randomly chosen nodes in a 1-D network can be readily obtained. Therefore, it is straightforward to extend the results obtained in this paper to 1-D networks with multiple source-destination pairs. Furthermore, in many dynamic networks, the density of nodes remains quite stable although the constituent nodes keep changing. For example, in vehicular networks, during peak hours and non-peak hours, the density of vehicles on a particular road segment is quite stable and readily predictable. However the particular vehicles on the road segment keep changing. Therefore, in this paper we assume that each node knows the density of the nodes in the network but not their number and exact locations.

As commonly done in the literature [2], [4], [5], [1], when we consider the transmission of a packet between a source and a destination, movement of nodes during the end-to-end transmission of the packet is not considered. That is, we consider a snap-shot of the network at a particular time instant. A typical end-to-end transmission can be completed in the order of milliseconds, during which the movement of nodes is comparatively small.

Furthermore, we consider that a pair of nodes are directly connected if and only if their Euclidean distance is smaller than or equal to $R$. That is, the well-known unit disk connection model is considered. The unit disk model is a simplified connection model that grossly captures the fact in wireless communications that it is easier for two nearby nodes to directly communicate with each other, compared with two nodes separated by a larger distance. Admittedly, connection model in real networks, 
particularly in vehicular networks, is more complicated than that presented in the unit disk model. Here the use of the unit disk model helps us to ignore the impact of physical layer details and focuses on the impact of the topology aspect of the network, which is the main focus of this paper.

We assume that each node knows its own location, this can be obtained easily either from an embedded GPS receiver, which is becoming increasingly ubiquitous in many mobile devices and vehicles [4] or via one of the numerous wireless localization techniques available [11], the location of the transmitter from which the node receives the packet of interest, the location of the destination, which can be carried in the packet header, and the node density. Using the above information, the node makes forwarding decision independently without prior coordination with its neighbors and even without being aware of their existence. More specifically, the following rule is used in making a forwarding decision when a node located at $x \in[0, L]$ overhears a packet:

- The node located at $x$ calculates the probability that it will forward the packet using its own location, the location of the transmitter, denoted by $z$, and the forwarding probability function $P_{f}$ according to $P_{f}(x-z)$. The design of the forwarding probability function $P_{f}$ will be explained later.

- If the node decides to forward the packet, it first waits a random backoff time $t$. The back-off time follows an exponential distribution [8] with mean $\frac{1}{\lambda}$. Then three situations may possibly occur: (1) if it does not overhear any transmission during the backoff period, it will forward the packet as the new transmitter; or (2) the node at $x$ overhears the transmission from a node located at $y$ AND $y \geq x$. Recall that the source is located at the origin and the destination is located at $L$. Therefore, in this situation, there is no need for the node at $x$ to forward the packet. The node simply drops the packet; or (3) the node at $x$ overhears the transmission from a node located at $y$ AND $y<x$. In this situation, the transmission by the node at $x$ may still help the packet to reach nodes that have not received the packet before. Therefore the node at $x$ updates its forwarding probability using the the node at $y$ as the new transmitter: $P_{f}(x-y)$, and makes decision on whether it will forward the packet according to $P_{f}(x-y)$, independently of its previous decision. If it decides to forward the packet, it starts a new backoff process. Otherwise, it gives up forwarding and discards the packet.

- The process naturally stops when the packet reaches its destination, which occurs when a node located in $[L-R, L)$ receives the packet and transmits.

The design of the uncoordinated cooperative forwarding scheme is intended to strike a balance among the amount of information and coordination required to make a forwarding decision, the transmission success probability and the number of transmissions (or equivalently forwarding nodes) required to reach the destination.

A node at $x$ is said to be a $k$-hop receiver if when the packet is received by the node for the first time, the

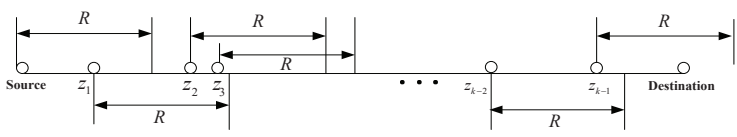

Figure 1. An illustration of the transmitters in a 1-D highly dynamic network

packet has been transmitted $k$ times by nodes located within $[0, x)$ including the transmission by the source. A node at $x$ is said to be a $k$-hop transmitter if by the time when the node transmits following the rules described in the last paragraph, the packet has already been transmitted $k$ times by nodes located within $[0, x)$ including the transmission by the source. Following the definition, the source is counted as the $0^{\text {th }}$ hop transmitter. Note that in our forwarding scheme, it is possible that a $k$-hop receiver is a $m$-hop transmitter for $m \geq k$. Let $z_{k}$ be the location of the $k$-hop transmitter. It follows that all nodes in $\left(z_{k-1}, z_{k-1}+R\right]$ are either $k$ hop receivers or $m$-hop receivers for $m<k$. Furthermore, for a fixed value of $k$, there can be at most one node that is a $k$-hop transmitter.

Let $\phi_{k}(x)$ be the probability that a node at $x$ is a $k$ hop receiver. Let $M(x)$ be the number of transmissions (or forwarding nodes) required to deliver the packet from the source to a node located at $x$ distance away using our uncoordinated forwarding scheme. $M(x)$ is a random positive integer. Let $P_{\text {suc }}(x)$ be the probability that a packet transmitted from the source successfully reaches a node at $x$. We note the following relationship that will be used in the latter analysis:

$$
P_{\text {suc }}(x)=\sum_{k=1}^{\infty} \phi_{k}(x) \text { and } E[M(x)]=\sum_{k=1}^{\infty} k \phi_{k}(x)
$$

Given the above definitions, the objective of the uncoordinated cooperative forwarding scheme design can be written analytically as follows:

$$
\begin{array}{cc}
\min _{P_{f}(x)} & E[M(L)] \\
\text { s.t. } & P_{\text {suc }}(L) \geq 1-\varepsilon
\end{array}
$$

where $\varepsilon$ is the a pre-designated small positive constant.

\section{Performance Analysis of the UNCOORDINATED COOPERATIVE FORWARDING SCHEME}

In this section, we analyze the performance of the uncoordinated cooperative forwarding scheme proposed in the last section where the performance is measured by two metrics: $P_{\text {suc }}(L)$ and $M(L)$. On the basis of the analysis, we then investigate the design of forwarding probability function $P_{f}(x)$ that minimizes the expected number of transmissions while meeting the performance objective that $P_{\text {suc }}(L) \geq 1-\varepsilon$.

Fig.1 illustrates the locations of the transmitters in the 1-D highly dynamic network, where $z_{k}$ is the location of the $k$-hop transmitter and $z_{0}=0$ is the location of the source.

Let $\varphi_{k}(x)$ be the probability that a node located at $x$ (if exists) is a $k$-hop transmitter. In the rest of this section, we 
first obtain an analytical expression of $\varphi_{k}(x)$ recursively for $k=1,2, \ldots$ On that basis, we obtain $\phi_{k}(x), P_{\text {suc }}(L)$ and $M(L)$.

Now we start with $k=1$. A node at $x$ is a 1-hop transmitter if and only if the following conditions are met:

- The node has received the packet from the source. This occurs with probability $g(x)$ where $g(x)$ is the connection function. For the unit disk model, $g(x)=$ 1 when $0<x \leq R$ and $g(x)=0$ otherwise.

- The destination has not received the packet. This occurs with probability $1-g(L)$.

- The node decides to forward the packet. This occurs with probability $P_{f}(x)$.

- The node is successful in the competition in backoff process and gets the opportunity to transmit. This occurs with probability $h$ depending on the backoff process only. The value of $h$ will be analyzed shortly later.

Noting that the above four events are independent, it follows that

$$
\varphi_{1}(x)=h g(x)(1-g(L)) P_{f}(x)
$$

Next we analyze $h$. Let $t_{x}^{*}$ be the (random) backoff timer of the node at $x$. Conditioned on $t_{x}^{*}=t$, the node at $x$ becomes successful in the backoff competition if all other nodes, who have received the packet from the source AND who decides to forward the packet, has a backoff timer greater than $t$. Note the independence between the event that a node receives the packet, the event that a node decides to forward the packet and the event that a node whose backoff timer is smaller than or equal to $t$. Denote by $\operatorname{Pr}\left(t^{*}<t\right)$ the probability of the event that a randomly chosen node whose backoff timer is smaller than or equal to $t$. Because the backoff timer follows an exponential distribution with mean $\frac{1}{\lambda}$, it readily follows that $\operatorname{Pr}\left(t^{*}<t\right)=1-e^{-\lambda t}$.

Using the thinning theorem [12], the set of nodes, who receives the packet AND decides to forward the packet AND has a backoff timer smaller than or equal to $t$, follows an inhomogeneous Poisson distribution with density $\rho g(x)(1-g(L)) P_{f}(x) \operatorname{Pr}\left(t^{*}<t\right)$. The number of such nodes follows an exponential distribution with mean $\int_{0}^{L} \rho g(x)(1-g(L)) P_{f}(x) \operatorname{Pr}\left(t^{*}<t\right) d x$. Therefore the probability that there is no such node is given by

$$
e^{-\int_{0}^{L} \rho g(x)(1-g(L)) P_{f}(x) \operatorname{Pr}\left(t^{*}<t\right) d x}
$$

Further using the Slivnyak-Mecke theorem [12], the conditional event that there exists a node at $x$ does not affect the above probability. Note that the probability density function (pdf) of $t^{*}$ is $\lambda e^{-\lambda t}$. Therefore using the total probability theorem

$$
h=\int_{0}^{\infty} e^{-\int_{0}^{L} \rho g(x)(1-g(L)) P_{f}(x)\left(1-e^{-\lambda t}\right) d x} \lambda e^{-\lambda t} d t
$$

Since in the unit disk model, $g(x)=0$ for $x>$ $R$, the above expression can also be written as $\int_{0}^{\infty} e^{-\int_{0}^{R} \rho g(x)(1-g(L)) P_{f}(x)\left(1-e^{-\lambda t}\right) d x} \lambda e^{-\lambda t} d t$. Combin- ing (3) and (4), it follows that

$$
\begin{aligned}
& \varphi_{1}(x)=g(x)(1-g(L)) P_{f}(x) \\
& \times \int_{0}^{\infty} e^{-\int_{0}^{R} \rho g(y) P_{f}(y)\left(1-e^{-\lambda t}\right) d y} \lambda e^{-\lambda t} d t
\end{aligned}
$$

Now we proceed to the case that $k$ takes more general values other than 1 . Let $z_{k}, k>1$ be the random location of the $k$-hop transmitter. Assuming that the pdf of $z_{k}$, i.e. $\varphi_{k}(x)$, is known. We shall derive the pdf of $z_{k+1}$ conditioned on that $z_{k}=y$ or simplify referred to as conditioned on $z_{k}$. Note that according to the design of our uncoordinated cooperative forwarding scheme, when the node at $z_{k}$ transmits as the $k$-th hop transmitter, the node essentially replaces the role of the source in the system. Therefore it readily follows that

$$
\begin{array}{r}
\varphi_{k+1}\left(x \mid z_{k}\right)=g\left(x-z_{k}\right)\left(1-g\left(L-z_{k}\right)\right) P_{f}\left(x-z_{k}\right) \\
\times \int_{0}^{\infty} e^{-\int_{z_{k}}^{z_{k}+R} \rho g\left(y-z_{k}\right) P_{f}\left(y-z_{k}\right)\left(1-e^{-\lambda t}\right) d y} \lambda e^{-\lambda t} d t
\end{array}
$$

Using the property that for a fixed value of $k$, there can be at most one node who is a $k$-hop transmitter, the unconditional probability can be obtained as

$$
\begin{aligned}
& \varphi_{k+1}(x)=\int_{0}^{L} \varphi_{k+1}\left(x \mid z_{k}\right) \varphi_{k}\left(z_{k}\right) \rho d z_{k} \\
= & \int_{0}^{L} \cdots \int_{0}^{L} \varphi_{k+1}\left(x \mid z_{k}\right) \\
\times & \varphi_{k}\left(z_{k} \mid z_{k-1}\right) \cdots \varphi_{2}\left(z_{2} \mid z_{1}\right) \varphi_{1}\left(z_{1}\right) \rho^{k} d z_{1} \cdots d z_{k}
\end{aligned}
$$

The destination is a $k$ hop receiver if and only if it can directly receive from a $k-1$ hop transmitter. Note that the term $1-g\left(L-z_{k}\right)$ in $\varphi_{k+1}\left(x \mid z_{k}\right)$ allows us to rule out the possibility that transmission continues after the packet has reached its destination. Further note that for any fixed value of $k$, there is at most one node that can be a $k$ hop transmitter. Based on the above observations, it follows that when $k>1$

$\phi_{k}(L)=\int_{0}^{L} \varphi_{k-1}(y) \rho g(L-y) d y=\int_{L-R}^{L} \varphi_{k-1}(y) \rho d y$

and $\phi_{1}(L)=g(L)$.

Equation (8), together with (1), allows us to determine the transmission success probability $P_{\text {suc }}(L)=$ $\sum_{k=1}^{\infty} \phi_{k}(L)$ and the expected number of transmissions required to reach the destination $E[M(L)]=\sum_{k=1}^{\infty} k \phi_{k}(x)$.

\section{A. Design of Optimal Forwarding Probability Function}

As manifested in (5), (6) and our discussion in Section I, the forwarding probability function $P_{f}(x)$ plays an important role in determining the performance of the forwarding scheme. Based on the analysis in the last section, in this subsection we analyze the design of the optimal forwarding probability function for the optimization problem in (2).

The analytical expressions for $P_{\text {suc }}(L)$ and $E[M(L)]$ in their present form do not allow us to readily analyze the optimal functional form of $P_{f}(x)$ that solves the optimization problem in (2). However, for particular forms of $P_{f}(x)$, e.g. $P_{f}(x)=c$ and $P_{f}(x)=a x$, the optimum 


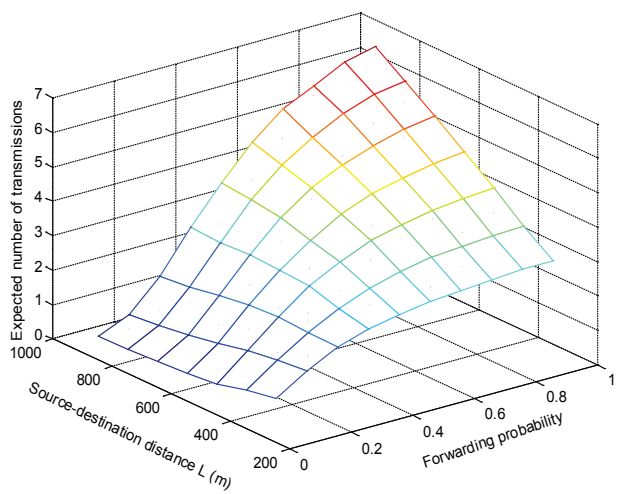

Figure 2. Expected number of transmissions as a function of $P_{f}(x)=c$ and $L(R=250 \mathrm{~m}, \rho=0.02 / \mathrm{m})$

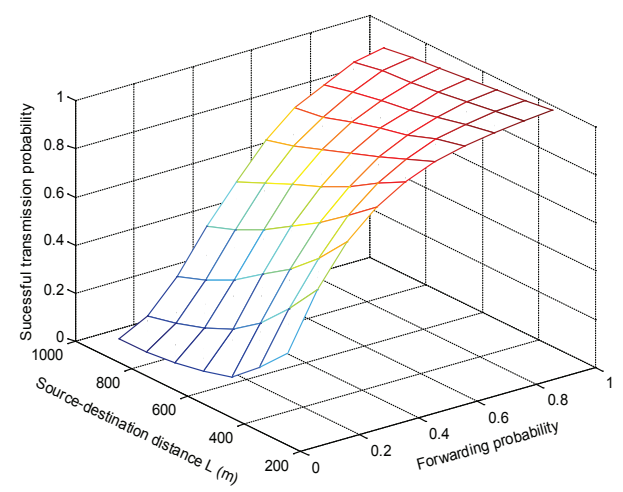

Figure 3. Successful transmission probability as a function of $P_{f}(x)=$ $c$ and $L(R=250 \mathrm{~m}, \rho=0.02 / \mathrm{m})$

parameters for $P_{f}(x)$ can be found using the method of Lagrange multipliers and solved numerically.

In the following analysis, we consider the simple case that $P_{f}(x)=c$ as an example. The optimization problem in (2). now reduces to finding the value of $c_{o p t}$ :

$$
\begin{array}{cc}
c_{\text {opt }}=\underset{P_{f}(x)=c}{\operatorname{argmin}} & E[M(L)] \\
\text { s.t. } & P_{\text {suc }}(L) \geq 1-\varepsilon
\end{array}
$$

The Lagrangian of the optimization problem can be written as

$$
\mathcal{L}\left(P_{f}(x)=c, \eta\right)=E[M(L)]+\eta\left(1-\varepsilon-P_{\text {suc }}(L)\right)
$$

The optimum value of $c$ can then be obtained by setting the partial derivative of $\mathcal{L}\left(P_{f}(x)=c, \eta\right)$ with regards to $c$ and with regards to $\eta$ to 0 respectively and solving the equations. However in this paper, we resort to a simpler method by using figures. Fig. 2 and 3 show the variation of $E[M(L)]$ and $P_{\text {suc }}(L)$ with the source-destination distance $L$ and the forwarding probability $P_{f}(x)=c$. Both figures are drawn using the analytical expression of $E[M(L)]$ and $P_{\text {suc }}(L)$. As shown in the figures, both $E[M(L)]$ and $P_{\text {suc }}(L)$ are non-decreasing function of $c$. Based on the observation, it follows that the optimum value of $c$ is the solution to the equation $P_{\text {suc }}(L)=1-\varepsilon$, which can be found numerically.

\section{Simulation}

In this section, we use both simulations and numerical results to establish the performance of the proposed uncoordinated cooperative forwarding scheme and provide some intuitively digestible results. Considering a 1D axis, nodes apart from source and destination are deployed following a homogeneous density $\rho=0.06 / \mathrm{m}$. The transmission range under unit disk model $R$ is $250 \mathrm{~m}$ [2]. The distance between source and destination $L$ varies from $250 \mathrm{~m}$ to $1000 \mathrm{~m}$. The back off time follows the exponential distribution with rate $\lambda=1$. The lower bound of successful transmission probability $P_{\text {suc }}^{\text {lower }}(L)$ is 0.95 . Note that each point in the simulation is the average value obtained from 50000 random simulations. The limit of the maximum number of hops $K_{m}$ is 20 .

Fig. 6 shows the expected number of transmissions as a function of the source-destination distance $L$ using both the constant forwarding probability $P_{f}(x)=0.34$ and the linear forwarding probability $P_{f}(x)=0.0025 x$. The parameter setting of the forwarding probability functions have been explained in the second paragraph of Section IV. It can be observed that under the same or similar successful transmission probability the uncoordinated cooperative forwarding scheme employing the linear forwarding probability requires less transmissions than that using a constant forwarding probability.

First, we evaluate the probability that the destination can be reached in $k$ transmissions assuming both constant forwarding probability $P_{f}(x)=c=0.34$ and linear forwarding probability $P_{f}(x)=a x=0.0025 x$ where $x \in[0, R]$. The value of $a$ and $c$ are determined assuming that $L=1000 \mathrm{~m}$ and $P_{\text {suc }}(L)=0.95$. Because the successful transmission probability increases with the decreasing $L$ which has been proved by Fig. 3, the successful transmission probability is undoubtedly not less than 0.95 when $L$ varies from $250 \mathrm{~m}$ to $1000 \mathrm{~m}$ conditioned on $P_{f}(x)=c=0.34$ (and $P_{f}(x)=a x=0.0025 x$ ). With the same or similar successful transmission probability $P_{\text {suc }}(L)$, we only need to compare the expected number of transmissions under two forwarding probability functions.

The probability that the destination can be reached in $k$ transmissions under two forwarding probability functions are shown in Fig. 4 and Fig. 5. The parameter

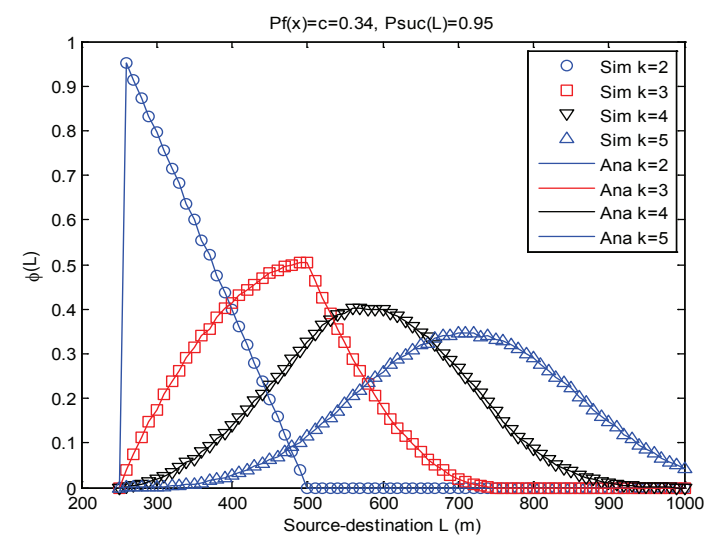

Figure 4. Probability that the destination at $L$ is a k-hop receiver $\left(P_{f}(x)=c=0.34\right)$. 


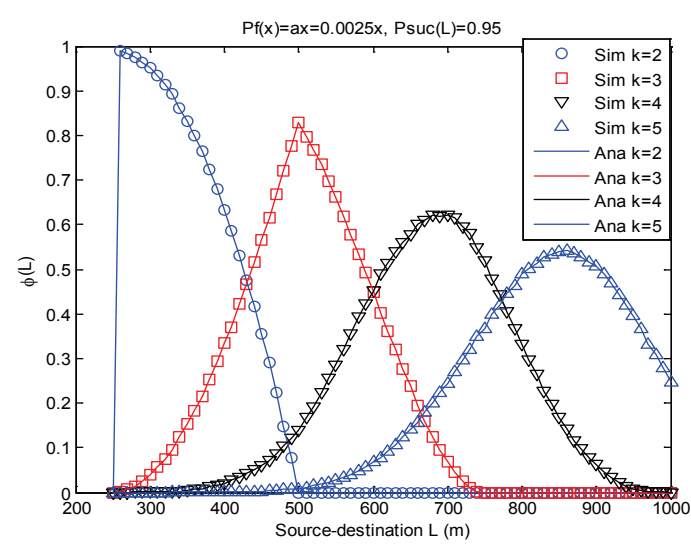

Figure 5. Probability that the destination at $L$ is a k-hop receiver $\left(P_{f}(x)=a x=0.0025 x\right)$.

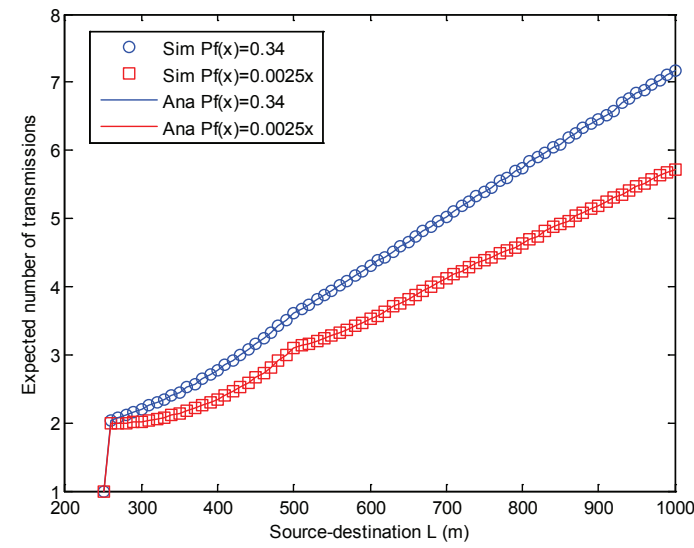

Figure 6. Expected number of transmissions.

setting of the forwarding probability functions have been explained in the second paragraph of Section IV. The confidence interval is too small to be distinguishable and hence ignored. Unsurprisingly, the numerical result and the simulation result match very well. A visual comparison of Fig. 4 and Fig. 5 appears to suggest that a less number of transmissions is required when using a linear forwarding probability $P_{f}(x)=0.0025 x$ compared with that using a constant forwarding probability $P_{f}(x)=0.34$. This result is consistent with our intuition that the linear forwarding probability results in the fact that the node which is farther away from the transmitter is in a better position to transmit to the destination and results in less number of total transmissions.

Fig. 8 compares the performance of uncoordinated cooperative forwarding schemes, measured in the expected number of transmissions $E[M(L)]$, using three different forwarding probability functions, i.e. $P_{f}(x)=c, P_{f}(x)=$ $a x$ and $P_{f}(x)=\left\{\begin{array}{ll}0, & 0 \leq x<b R \\ 1, & b R \leq x \leq 1\end{array}\right.$. In the figure, the three cases are labeled as uncoordinated Case 1, uncoordinated Case 2 and uncoordinated Case 3 respectively. For comparison, we also provide the expected number of transmissions using the shortest path routing. Obviously, the shortest path routing needs global knowledge of the network and it serves as a benchmark for the best performance here. This is labeled as Case 4 in Fig. 8.
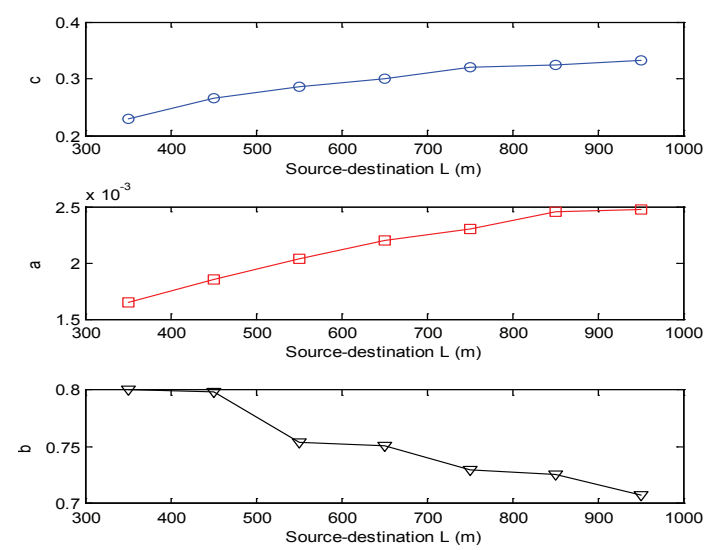

Figure 7. Optimal value of $c, a$ and $b$ conditioned on $P_{\text {suc }}(L)=1-\varepsilon=$ 0.95 .

Fig. 7 shows the optimal value of $c, a$ and $b$ corresponding to the different values of $L$ under the constraint that $P_{\text {suc }}(L)=1-\varepsilon=0.95$, which justifies that the forwarding probability should be higher with an increasing value of $L$ in order to guarantee a high successful transmission probability. Interestingly, Case 3 where $P_{f}(x)$ is set as a step function provides the best performance among all three cases, close to that of Case 4 (a benchmark for the best performance), as shown in Fig.8. It is part of our future work to dig into the findings and provide analytical support for the optimum choice of $P_{f}(x)$.

Fig. 9 compares the expected number of transmissions $E[M(L)]$ of the proposed uncoordinated forwarding scheme and the coordinated greedy forwarding algorithm [13]. The greedy forwarding algorithm chooses the node, which has received a copy of the packet and would like to forward, closest to the destination in each hop as the forwarding node. Greedy forwarding algorithm is comparatively easy to implement however relies on coordination between nodes to determine the set of nodes who have received packet and would like to forward, and to determine which node among them is closest to the destination. The three sub-figures compare the performance of the proposed uncoordinated scheme and the greedy algorithm, using three different forwarding probability functions as mentioned above, i.e. $P_{f}(x)=c$ (Case 1), $P_{f}(x)=a x$ (Case 2) and $P_{f}(x)= \begin{cases}0, & 0 \leq x<b R \\ 1, & b R \leq x \leq 1\end{cases}$ (Case 3) respectively. From Fig. 9, we can see that the proposed uncoordinated scheme has a similar performance as the greedy forwarding algorithm but saving a large amount of coordination overhead.

\section{CONCLUSION AND FUTURE WORK}

This paper proposed an uncoordinated cooperative forwarding scheme for 1-D highly dynamic networks. The performance of the proposed scheme, measured in terms of the transmission success probability and the expected number of transmissions, is analyzed. On that basis, design of the optimum forwarding probability function is discussed. Given a particular form of the forwarding proba- 


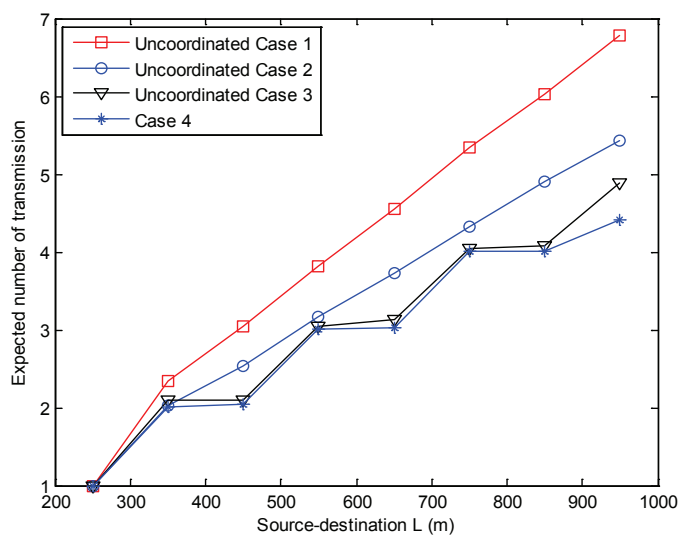

Figure 8. Expected number of transmissions using three forwarding probability functions

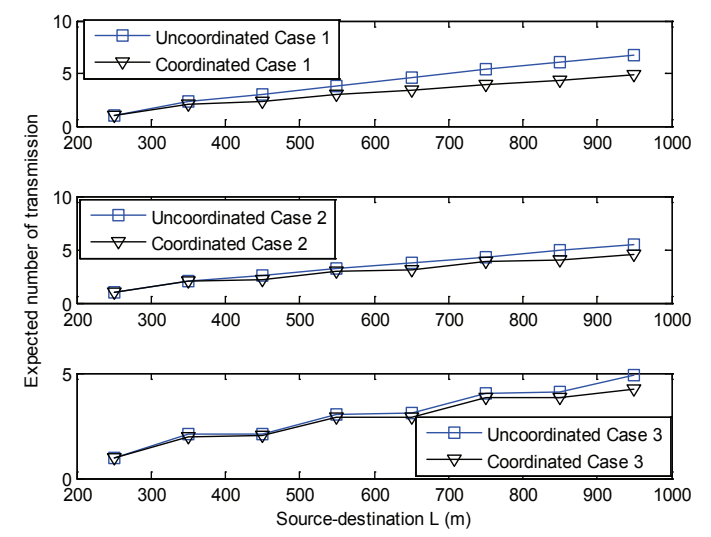

Figure 9. A comparison of expected number of transmissions using the proposed uncoordinated schemes and using coordinated greedy forwarding scheme under three forwarding probability functions

bility function, our analysis can be used to numerically determine the optimum parameter settings for the forwarding probability function that minimizes the expected number of transmissions while meeting the performance target on the transmission success probability. Furthermore, the performance of the uncoordinated cooperative forwarding scheme employing three commonly used forwarding probability functions, i.e., the constant forwarding probability, the linear forwarding probability and the step function forwarding probability, are compared. Our preliminary study appears to suggest that by choosing the forwarding probability function to be a step function, the best performance can be achieved. This finding is consistent with our earlier work in [4] studying a two-hop scenario in two-dimensional networks where the source and the destination is separated by at most two hops. It is part of our future work plan to dig into the result and provide analytical support for the optimum choice of $P_{f}(x)$.

\section{ACKNOWLEDGMENT}

The Project was supported by the Joint Research Fund for Overseas Natural Science of China (Grant No. 61428102), the Science and Technology Cooperation Project of Hong Kong, Macao and Taiwan (Grant No. 2014DFT10320A) and Beijing Nova Program(no. xx2012037).

\section{REFERENCES}

[1] J. Andrews, S. Shakkottai, R. Heath, N. Jindal, M. Haenggi, R. Berry, G. Dongning, M. Neely, S. Weber, S. Jafar, and A. Yener, "Rethinking information theory for mobile ad hoc networks," Communications Magazine, IEEE, vol. 46, no. 12, pp. 94-101, 2008.

[2] S. Panichpapiboon and C. Lin, "Irresponsible forwarding under real intervehicle spacing distributions," Vehicular Technology, IEEE Transactions on, vol. 62, no. 5, pp. 2264-2272, 2013.

[3] G. Mao, "Research on wireless multi-hop networks: Current state and challenges," Journal of Communications, Special Issue on Future Directions in Computing and Networking, vol. 7, no. 5, pp. 357-364, 2012.

[4] X. Lixiang, L. Libman, and M. Guoqiang, "Uncoordinated cooperative communications in highly dynamic wireless networks," Selected Areas in Communications, IEEE Journal on, vol. 30, no. 2, pp. 280-288, 2012.

[5] Z. Chao, Z. Wei, and M. Guoqiang, "Uncoordinated cooperative communications with spatially random relays," Wireless Communications, IEEE Transactions on, vol. 11, no. 9, pp. 3126-3135, 2012.

[6] S. Panichpapiboon and W. Pattara-Atikom, "A review of information dissemination protocols for vehicular ad hoc networks," Communications Surveys Tutorials, IEEE, vol. 14, no. 3, pp. 784798, 2012.

[7] S. Dulman, M. Rossi, P. Havinga, and M. Zorzi, "On the hop count statistics for randomly deployed wireless sensor networks," Int. J. Sen. Netw., vol. 1, no. 1/2, pp. 89-102, 2006.

[8] S. Biswas and R. Morris, "Exor: opportunistic multi-hop routing for wireless networks," SIGCOMM Comput. Commun. Rev., vol. 35, no. 4, pp. 133-144, 2005.

[9] Z. J. Haas, J. Y. Halpern, and L. Li, "Gossip-based ad hoc routing," Networking, IEEE/ACM Transactions on, vol. 14, no. 3, pp. 479491, 2006.

[10] K. Jae-soo, Z. Qi, and D. P. Agrawal, "Probabilistic broadcasting based on coverage area and neighbor confirmation in mobile ad hoc networks," in Global Telecommunications Conference Workshops, 2004. GlobeCom Workshops 2004. IEEE, pp. 96-101.

[11] G. Mao, B. Fidan, and B. D. O. Anderson, "Wireless sensor network localization techniques," Computer Networks, vol. 51, no. 10, pp. 2529-2553, 2007.

[12] F. B. Blaszczyszyn and B., Stochastic Geometry and Wireless Networks, Volume I - Theory. Now Publishers Inc, 2009.

[13] M. B. Fragkiskos Papadopoulos, Dmitri Krioukov and A. Vahdat, "Greedy forwarding in dynamic scale-free networks embedded in hyperbolic metric spaces," pp. 2973-2981, March 14-19 2010. 